\title{
Stellar Acoustic Ray Patterns
}

\author{
J. Perdang \\ Institute of Astronomy \\ Madingley Road \\ Cambridge CB3 OHA, UK \\ and \\ Institut d'Astrophysique (*) \\ 5, Avenue de Cointe \\ Cointe-Ougree B-4200, Belgium \\ (*) permanent address
}

\begin{abstract}
We have analysed and attempted to classify the acoustic ray patterns in stars whose equilibrium figure is globally deformed. We discuss here the effect of distortions of ellipsoidal and pear-shaped structure. Under ellipsoidal symmetry all ray patterns remain 'regular'. For arbitrarily small pear-shaped deformations our numerical results indicate the occurrence of 'chaotic' ray patterns arising out of the unstable longest diameter ray. These chaotic rays cover the whole configuration space.
\end{abstract}

\section{Introduction}

In current inverse helioseismological problems information on the central parts of the sun $\left(r<.2 R_{\odot}\right)$ is very hard to extract from the frequency spectrum (cf Gough 1986). A major reason for this difficulty is that acoustic waves, except if strictly radial, do not penetrate into the central region of a star; the precise central properties of the sun have therefore only a minor effect on the global acoustic spectrum. One motivation of the present work is to see whether a similar difficulty survives if we relax the assumption of spherical symmetry of the star. To this end we investigate a class of formal models in which the surfaces of constant sound speed are algebraically deformed (Eq. 1). We then study the acoustic ray patterns arising under these conditions. The ray patterns directly indicate the zones avoided by the associated acoustic waves.

While the 'probability of avoidance' of the centre in a spherically symmetric star is always one - the set of radial rays is of measure zero among the totality of rays - this probability decreases as we consider ellipsoidal deformations. Moreover, as soon as we include pear-shaped deformations, we observe chaotic rays for initial conditions close to the longest diameter ray of the ellipse. These nontrivial results are indicative that in stars lacking the property of exact spherical symmetry the physics of the central region influence the global structure of the acoustic spectrum. The sensitivity of the spectrum to the central physics is increased as the configuration deviates more and more from the spherical symmetry.

\section{Ray Chaos}

An unsuspected result is the presence of 'observable' chaotic behaviour as soon as the ray equations (Eqs. 5) cease to be 'integrable', integrability being lost under an arbitrarily small pear-shaped distortion of the stellar structure. This result differs radically from the behaviour of the more conventional generic Hamiltonian systems discussed in the literature. The latter typically exhibit 'observable' chaotic behaviour only beyond some finite distortion of the Hamiltonian away from a reference integrable Hamiltonian, in accordance with the KAM theorem. In stellar ray problems KAM theory is not applicable, since the ray Hamiltonian violates the requirement of analyticity in the generalised coordinates and momenta (Perdang 1986). However, our numerical results indicate that the chaotic rays which are initially close to regular rays remain so over a long integration time, developing blurred or 'vague' caustics which in turn are the boundaries of 'vague' tori (Figs. 2). A similar behaviour has been reported in classical billiards (Berry 1981). It is also consistent with a class of solutions of analytic Hamiltonians (Shirts and Reinhardt 1982). The latter class of chaotic rays, which we may conveniently refer to as 'vague-regular' rays, coincide with the totality of the chaotic rays arising in slightly globally deformed stars such as the sun. This guarantees that we can apply the standard semi-classical quantisation 
method to the average 'vague' tori carrying these rays, in order to compute the asymptotic frequencies. Alternatively it suggests, although this point requires closer checking, that a standard perturbation method of the oscillation eigenvalue problem of the spherical star should provide an acceptable approximation to the actual eigenfrequencies of the slightly deformed star.

\section{Ray Equations and Model}

We investigate stars in which the originally spherical surfaces of constant sound speed are distorted into algebraic surfaces of third degree in the coordinates, of equation

$$
\sum_{i} a_{i} x_{i}^{2}+B \sum_{i j k} b_{i j k} x_{i} x_{j} x_{k}=r^{2}, i, j, k=1,2,3 ;
$$

$x_{1}, x_{2}, x_{3}$ are dimensionless coordinates, scaled such that the volume of the star is equal to $4 / 3 \pi$. The factor $r, 0 \leq r \leq 1$, is an averaged dimensionless radius. The ordering parameter $B$ is less than 1 , while the remaining coefficients $a$ and $b$ are of order 1 . Deformations in the surfaces of constant sound speed of form (1) are expected to arise generically under the action of any global perturbation of low enough symmetry.

We have examined the distortion due to the tidal effect of a mass point $M^{\prime}$ on the $x_{1}$ axis, at a distance $D$ from the centre of the primary; the latter is a liquid star considered in the Jeans approximation (Chandrasekhar 1969); the novel feature is that we expand the tidal potential to cubic degree in the coordinates

$$
V_{T}(x)=\mu\left\{x_{1}^{2}-1 / 2\left(x_{2}{ }^{2}+x_{3}^{2}\right)+(1 / D) x_{1}\left(x_{1}^{2}-3 / 2\left(x_{2}{ }^{2}+x_{3}^{2}\right)\right)\right\}, \mu=G^{\prime} / D^{3} .2
$$

Due to the symmetry of the perturbing potential (2), the coefficients in Eq. (1) obey

$$
a_{2}=a_{3} \text { and } b_{122}=b_{133}=-3 / 2 b_{111} \text {, }
$$

the remaining coefficients $b$ vanish. By setting $b_{111}=1$, the family of nested surfaces (1) labelled by $r$ is fixed by two parameters which we may take as $a_{1}$ and $B$. The condition of hydrostatic equilibrium relates these parameters to $\mu$ (a function of the excentricity and hence of $a_{1}$, cf Chandrasekhar 1969), and $1 / D=B / a_{1}$ (Moray 1986). The sound speed $c(x)$ then becomes

$$
c(x)^{2}=\gamma P(x) / \rho=c_{c}^{2}\left\{1-r^{2}(x)\right\},
$$

where $\gamma$ is as usual the (constant) adiabatic index, $P(x)$ is the pressure field, $\rho$ the (uniform) density and $c_{c}$ the central sound speed. The latter is normalised to 1 , which determines in turn a dimensionless time.

The acoustic ray patterns are obtained by solving the Hamiltonian equations

$$
\mathrm{d} / \mathrm{dt} \mathbf{x}=\partial / \partial \mathbf{k} \Omega ; \mathrm{d} / \mathrm{dt} \mathbf{k}=-\partial / \partial \mathbf{x} \Omega ; \text { with } \Omega(\mathbf{x}, \mathbf{k})=\mathrm{c}(\mathbf{x})|\mathbf{k}| \quad, \quad 5
$$

(k, wavevector, $\Omega$, frequency). The latter are integrated numerically in the interior of the star, and analytically in the neighbourhood of the surface, where the equations are singular; the analytic solution takes care of the reflection of the ray. We have studied ray systems confined to the symmetry planes of the configuration only (say the plane $x_{1}-x_{2}$ ). Since any ray is bound to cross the $x_{1}$ axis, we generate the totality of rays by the 3-parameter family of initial conditions $x_{1}=X, x_{2}=0,|k|=k$, and the angle $w$ of the wavevector with the $x_{1}$ axis.

\section{Classifying the Rays}

$\beta=0$ : Ellipsoidal Configurations

One can show, by the procedure adopted in Perdang (1986) for spherical symmetry, that the ray problem remains regular. The integration of Eqs. (5) disclose in fact 3 families of regular rays (Fig. 1.a,b,c). 

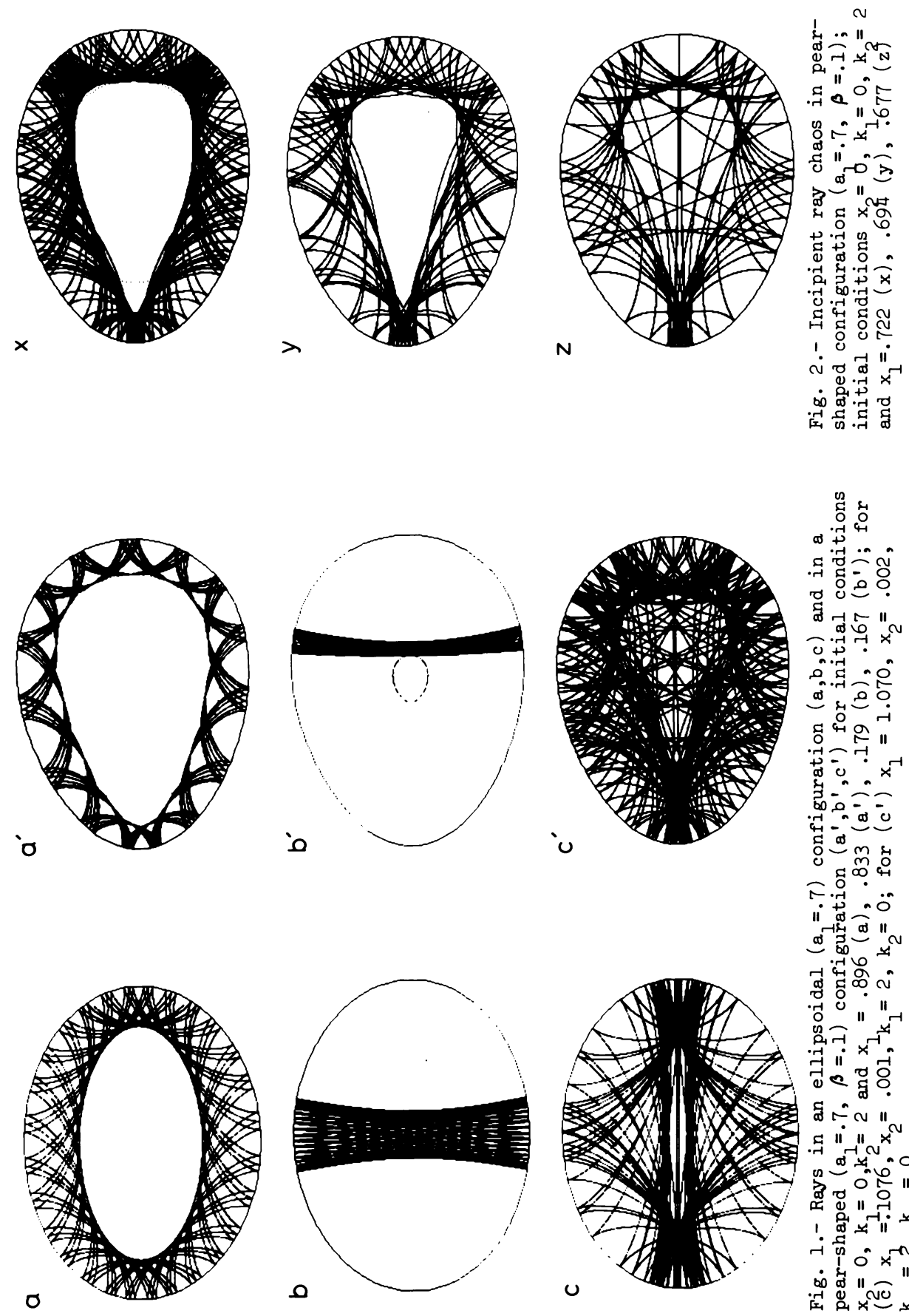

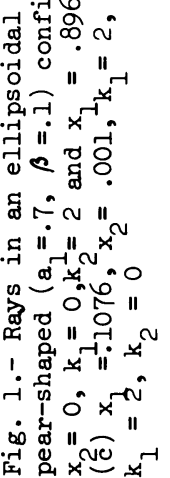


(a) Surface Rays (SRs) which avoid the central zones of the star (Fig. 1.a); the caustics of SRs of same frequency seemingly define a 1-parameter family of ellipses.

(b) Minor Axis Rays (MARs) which avoid the polar caps on the $\mathrm{x}_{1}$ axis (Fig. 1.b); the caustics of MARs of same frequency seem to define a 1-parameter family of hyperbolas.

(c) Focal Rays (FRs) are rays going through two foci, $x_{1}= \pm F$, on the $x_{1}$ axis (Fig. 1.c); FRs are the common limiting rays of both the SRs and MARs as the caustics of the latter become degenerate; we can view the caustic of the FRs as a degenerate ellipse (line segment on the $x_{1}$ axis joining $-F$ to $+F$ ) or as a degenerate hyperbola (longest diameter with the segment $-F$ to $+F$ being excluded).

As the ellipse transforms into a circle ( $F$ coincides with the origin) the region in the 3 dimensional parameter space $(X, k, w)$ producing $S R$ s coincides with this whole space, with the exception of the subset of dimension $2, X=0$. Only the latter subset produces MARs and FRs. For nonzero excentricity the subset carrying MARs becomes 3 dimensional, corresponding to $-F<X<F$, while $F R$ s correspond to the subsets $X= \pm F$, which remain 2 dimensional in the parameter space. This result shows that for any frequency there is indeed a 3 dimensional subset in the parameter space generating rays traversing the centre of the star, as soon as the ellipticity is nonzero. Incidentally, this explains also why for small elliptic deformations the chances of hitting a MAR remain small, inspite of the fact that MARs covering all of the symmetry plane do exist.

B = 0 : Pear-shaped Configurations

The ray problem now ceases to be integrable. Numerical integrations performed by choosing initial conditions in the parameter space $(X, k, w)$ demonstrate the existence of 3 families of rays directly related to the families of the ellipsoidal case (Figs. 1. $\left.a^{\prime}, b^{\prime}, c^{\prime}\right)$.

(a') SRs (Fig. 1.a') and (b') MARs (Fig. 1.b') are again regular classes of rays with caustics which are ellipses distorted into drop shapes, and deformed hyperbolas respectively. SRs are numerically found to survive until the caustic develops a cusp, say at $x_{1}=-C$ (closest to the point of greatest curvature of the surface). MARs show a larger avoided zone towards the side of greatest curvature of the surface.

(c') The limiting families of SRs (ray systems with cusped caustic) and of MARs (ray systems covering all of the region towards the smallest curvature, but showing an avoided zone near the greatest curvature of the surface) now cease to coincide. In the parameter space $(X, k, w)$ these limiting families define a 3 dimensional region $\mathrm{K}$ occupied by Chaotic Rays (CRs, Fig. 1.c'). That these rays are chaotic in the technical sense of the word can be verified by a numerical stability test. Notice that this class comprises the longest diameter ray (LDR) whose instability is geometrically obvious. For any nonzero value of $B$ the region $\mathrm{K}$ in the parameter space remains 3 dimensional, so that we have a nonzero probability of hitting a chaotic ray. Notice also that the CRs travel all through the star.

Our results show that although under arbitrarily slight generic distortions of the geometry of the star we always find chaotic rays, the corresponding chaos is of a mild form. More generally, these rays seemingly remain on tori over a long period of time if we choose initial conditions close to the regular regions. This is demonstrated in the examples exhibited in Fig. 2 which displays one (probably) SR (Fig. 2.x) and two nearby CRs (Figs. 2.y,z). In the latter cases a 'vague' caustic is found to survive which is indicative that the rays continue to stay on an approximate, or 'vague' torus (cf also Fig. 1.c').

\section{References}

Berry M.V. 1981 European J. Phys. 291

Chandrasekhar S. 1969 'Ellipsoidal Figures of Equilibrium', Yale University Press, New Haven

Gough D.O. 1986 in 'Seismology of the Sun and the Distant Stars' 125-140 D.O. Gough (ed), Reidel, Dordrecht

Moray F. 1986 Mémoire de licence, Université de Liège

Perdang J. 1986 in 'Seismology of the Sun and the Distant Stars' 141-171 D.O. Gough (ed), Reidel, Dordrecht

Shirts R.B., Reinhardt W.P. 1982 J. Chem. Phys. 775204 